\section{Cahiers de Narratologie}

Analyse et théorie narratives

$31 \mid 2016$

Sérialité narrative. Enjeux esthétiques et économiques

\title{
Journalisme narratif : des enjeux contextuels à la poétique du récit
}

\section{Marie Vanoost}

\section{(2) OpenEdition \\ Journals}

\section{Electronic version}

URL: http://journals.openedition.org/narratologie/7543

DOI: $10.4000 /$ narratologie. 7543

ISSN: $1765-307 X$

Publisher

LIRCES

\section{Electronic reference}

Marie Vanoost, « Journalisme narratif : des enjeux contextuels à la poétique du récit », Cahiers de Narratologie [Online], 31 | 2016, Online since 22 December 2016, connection on 21 December 2020. URL : http://journals.openedition.org/narratologie/7543; DOI : https://doi.org/10.4000/narratologie. 7543

This text was automatically generated on 21 December 2020.

Article L.111-1 du Code de la propriété intellectuelle. 


\title{
Journalisme narratif : des enjeux contextuels à la poétique du récit
}

\author{
Marie Vanoost
}

1 Le journalisme narratif est généralement défini comme une forme de journalisme « qui se lit comme un roman » (Wolfe, 1975, p. 21 ${ }^{1}$ ), mêlant la mission d'information publique du premier et les techniques d'écriture du second. Le journalisme factuel "classique», poursuivant une visée fondamentalement configurante ${ }^{2}$, s'organise selon le modèle de la pyramide inversée : il divulgue les informations les plus importantes dès les premières lignes de l'article pour offrir au lecteur, d'emblée, les clés de compréhension de l'information. Comme l'écrit Raphaël Baroni, selon ce modèle, l'article de presse

écrase la temporalité, [...] privilégie l'élaboration d'une explication du geste au détriment de la production d'une tension qui serait orientée vers un dénouement incertain. Ce que cherche à reconstruire le journaliste, c'est une cohérence permettant de relier l'événement récent à la totalité d'un vécu ou d'un état de la société. (Baroni, 2009, p. 77)

2 Tout en revendiquant la même visée configurante ${ }^{3}$, le journalisme narratif adopte la forme et les procédés textuels des récits intrigants ${ }^{4}$, jouant sur la tension d'un dénouement incertain qui repousse - au moins - certaines des clés de compréhension à la fin de l'article (Vanoost, 2013a). Selon la formule de Jon Franklin, auteur d'un manuel de journalisme narratif outre-Atlantique, une histoire est une séquence d'actions qui se produit lorsqu'un personnage sympathique rencontre une complication qu'il affronte, puis résout (Franklin, 2002, p. 71).

3 Si plusieurs auteurs et praticiens considèrent alors le journalisme narratif comme un genre - au même titre que le reportage ou l'éditorial, par exemple -, défini par l'adoption d'une forme narrative intrigante, cette position nous semble doublement problématique. D'une part, alors qu'il existe un certain nombre de genres journalistiques déjà établis, le journalisme narratif ne s'applique pas forcément à toutes les productions d'un même genre - ainsi, tous les reportages, par exemple, ne prennent pas la forme d'un récit intrigant. D'autre part, le journalisme narratif transcende les frontières existantes pour 
s'appliquer à différents genres - outre des reportages, on peut également trouver, notamment, des portraits ou des enquêtes narratifs.

Il nous semble dès lors plus pertinent de parler, à la suite de Michael Schudson (1978), de modèle. Un modèle journalistique, selon la conception de Schudson, est guidé par un idéal qui définit le rôle des journalistes et des médias qui y adhèrent, mais qui peut être approché de diverses façons et à des degrés divers. La notion de modèle renvoie ainsi à une forme relativement flexible de sérialité de type générique, mais qui relève d'un niveau supérieur aux genres journalistiques établis. Alors que l'idéal qui guide le journalisme factuel «classique » est celui de l'information objective et largement désincarnée, l'idéal qui guide le modèle journalistique narratif apparaît comme celui d'un récit largement similaire au récit canonique défini par la narratologie à partir de la fiction (Vanoost, 2013b).

5 Cet idéal alternatif par rapport au modèle dominant transforme, déplace, renouvelle les pratiques des journalistes et des médias qui mettent en œuvre le modèle narratif, dessinant ainsi un ensemble d'enjeux éthiques, économiques et professionnels propres au journalisme narratif. Dans un premier temps, cet article s'attache à esquisser les grandes lignes de ces différents types d'enjeux, tout en soulignant à quel point ils sont interdépendants et interconnectés, ne pouvant être envisagés que dans un réseau complexe. Cette première partie s'appuie sur le discours de vingt-cinq praticiens du modèle narratif, rencontrés en entretiens ${ }^{5}$ : quatorze reporters et éditeurs américains, travaillant principalement au sein de quotidiens et plus marginalement de quelques magazines, et onze journalistes et responsables de publication français et suisses, travaillant majoritairement pour des revues - souvent appelées mooks ${ }^{6}$ - mais aussi pour quelques journaux.

6 Ensuite, dans un second temps, l'article se propose d'explorer, à partir de quelques exemples tirés des récits des journalistes rencontrés en entretiens, comment cet enchevêtrement d'enjeux éthiques, économiques et professionnels influence la poétique des récits journalistiques et donne naissance à des innovations formelles permettant de réconcilier les fonctions narratives intrigante et configurante (Baroni, 2009), d'offrir une expérience de lecture proche de celle d'un récit fictionnel tout en rassurant le lecteur sur le caractère réel et vérifié des événements racontés.

\section{Discours des enjeux, enjeux des discours}

\section{"Bénéfices » et « dangers » du récit ${ }^{7}$}

7 Lorsqu'on les interroge sur leurs pratiques, les journalistes qui mettent en œuvre le modèle narratif avancent principalement des arguments d'ordre éthique pour justifier et promouvoir le journalisme narratif. Ils sont largement persuadés, tout d'abord, qu'en optant pour une forme de récit intrigant, ils peuvent intéresser le lecteur à des articles qu'il n'aurait peut-être pas lus, ou pas lus jusqu'au bout, sous une autre forme. Comme le dit Jeff Klinkenberg8,

A lot of stories, I call it, they have broccoli. Broccoli is something that's good for you. Eat it. Maybe you don't like it but you should eat it, it's good for you. And sometimes in a story that may be something technical, a bunch of numbers, or science, or whatever. And the readers, really, they don't want to read that, they lose patience. [...] you also need ice cream cones to make the broccoli tolerable. And so 
those ice cream cones become the force that's gonna drive readers through that story 9 . œuvre, peut également poser question sur un plan éthique. Comme l'explique Anne Hull ${ }^{12}$ , l'idée même d'une mise en intrigue de l'information s'oppose à la façon dont les journalistes envisagent généralement leur mission :

I think one of the biggest ethical dilemmas with this kind of writing is withholding information from the reader and you're sort of manipulative by putting the information at the end or the resolution. That in a way is the opposite to everything journalists kind of grow up to be taught. And I don't really have an answer for that. I've done it myself. Yeah, we just do it, I do it on almost every big story because there has to be a reason the reader keeps going ${ }^{13}$.

En outre, même si les praticiens rencontrés soutiennent que le modèle narratif permet une meilleure compréhension de l'information, ils sont loin de penser que toute information devrait prendre la forme d'un récit. Au-delà des cas où le modèle narratif ne semble pas approprié - comme lorsqu'il s'agit d'information dite "chaude ", de dernière minute -, Tom French ${ }^{14}$ explique que, précisément parce que le récit demande plus d'engagement de la part du lecteur, il ne peut pas être le seul modèle utilisé : si tous les articles demandaient autant d'engagement de la part du lecteur, celui-ci s'épuiserait.

Enfin, comme c'était déjà le cas concernant les « bénéfices » éthiques du modèle narratif, la réflexion des praticiens dépasse le cadre restreint de l'éthique journalistique pour 
embrasser, de manière plus fondamentale, le rôle du journaliste en tant que conteur. Ainsi, pour Jacqui Banaszynski :

In some ways we might be wired [...] a little too much for stories because stories can so completely shape and influence, again, how we view history. The storytellers are the ones who own our vision of history. This all notion that if you own the right to tell the story, you kind of own the truth, wow, that's kind of huge $e^{15}$.

\section{De l'éthique à l'économique}

Toutes ces considérations éthiques, y compris les plus désintéressées et les moins prosaïques, s'avèrent néanmoins indissociables d'enjeux d'ordre économique. Cette articulation apparaît particulièrement clairement lorsque, interrogé sur les raisons pour lesquelles il s'est tourné vers le modèle journalistique narratif, Eli Saslow ${ }^{16}$ évoque, en seulement trois phrases, des éléments qui renvoient tant à la survie de la presse écrite qu'à l'objectif de compréhension profonde du journalisme narratif :

I think part of it is it's important as a self-preservation technique for writers and newspapers. I think you have to make people care to be invested in the things that you're writing about. But I also think that there is no better way to understand what people are going through and what people's lives are like. ${ }^{17}$

Le journalisme et, particulièrement, la presse écrite sont en effet actuellement en profonde crise : concurrence avec les médias en ligne, baisse des chiffres de vente, perte de confiance des lecteurs, etc. Dans ce contexte, le journalisme narratif est considéré par nombre de ses praticiens comme une - ou, pour certains même, la - solution. C'est le cas notamment de John Woodrow $\operatorname{Cox}^{18}:$ «I think it's really kind of the only way... or it's one way that we'll survive. You know, I think newspapers have to add value to people's lives and one way to do that is to give them really good stories to read $^{19}$. » $\mathrm{Ou}$, selon les termes de Léna Mauger ${ }^{20}:$ «Je crois [...] qu'aujourd'hui la plupart des journaux ne font plus envie, pour des tas de raisons, et que notre rôle aujourd'hui de journaliste c'est encore de donner envie aux gens de s'informer sur le monde. »

Fondamentalement, capter l'intérêt du lecteur est un enjeu économique de base pour tout média d'information. Pour y parvenir, les techniques narratives intrigantes apparaissent comme un outil particulièrement intéressant - surtout pour les interviewés américains. Comme l'explique, par exemple, Lane DeGregory ${ }^{21}$ :

When they [the editors] get the feature page, they start coming to me and say "you have six inches on the front." So I say "okay, at the end of the six inches I have to have something good, you know, a question so that you have to turn that page." I really do think about that and I think of that like planting breadcrumbs, you know, that you want someone to pick them up and follow in your story ${ }^{22}$.

17 Attirer le lecteur dans un récit ne suffit néanmoins sans doute pas à assurer la fidélité de ce lecteur. Il semble également nécessaire de convaincre le lecteur qu'on ne lui offre pas seulement une valeur ajoutée en termes de plaisir de lecture, mais également une valeur ajoutée par rapport à ce qui constitue la mission première du journalisme : l'information. Selon Adrien Bosc ${ }^{23}$,

Il y a une attente, que moi souvent je définis comme une sorte de paradoxe nécessaire, qu'à la gratuité, qu'à la dématérialisation de l'information, répond finalement un besoin concomitant et paradoxal de lenteur, de prise de position et d'information qu'on ne trouve nulle part ailleurs, et que seuls ceux qui auront ces trois points vraiment chevillés au corps, dans leur publication, instaureront un rapport de confiance avec le lecteur, et donc le feront revenir à chaque numéro. 
18

mbreux interviewés francophones insistent à cet égard sur le succès des mooks ${ }^{24}$ et sur le retour de formats plus longs dans la presse quotidienne de qualité - Le Monde et Libération, principalement. Les interviewés américains soulignent quant à eux que plusieurs journaux font le pari d'intégrer à leur rédaction une équipe de journalistes spécialistes du format narratif - c'est le cas du Washington Post et du Tampa Bay Times -, tandis que d'autres rédactions - comme celle du New York Times, par exemple - comptent l'un ou l'autre praticien du modèle. Ils se montrent néanmoins globalement moins optimistes que les interviewés francophones quant à l'avenir du journalisme narratif dans leur propre média. Le problème étant - des deux côtés de l'Atlantique d'ailleurs - que ce type de journalisme est - ou, à tout le moins, est perçu - comme coûteux pour les rédactions. Cependant, pour Tom French, «I think it has to do with really sort of helping people open up their definition of what narrative is. A lot of editors, a lot of writers think that narrative is just writing massive long feature stories ${ }^{25}$. »

\section{En toile de fond, l'identité professionnelle}

Pour les médias et les journalistes qui optent pour le journalisme narratif, il s'agit donc aussi de définir une identité professionnelle particulière. Le discours éthique tenu par les interviewés pour justifier et promouvoir le modèle narratif participe bien entendu à cette recherche et cette affirmation identitaire : déclarer intéresser les lecteurs autrement et leur proposer une autre forme de compréhension de l'information, c'est forcément aussi se positionner au sein du champ journalistique. Il y a ainsi d'autres enjeux derrière les enjeux que les journalistes identifient dans leur discours éthique.

De la même manière, on peut difficilement séparer les enjeux économiques des logiques de positionnement professionnel. C'est particulièrement frappant en ce qui concerne les mooks et leur modèle économique volontairement décalé, qui contribue largement à leur positionnement à mi-chemin entre les sphères journalistique et littéraire. Pour autant, tout en affichant une volonté claire de se distinguer de la presse "traditionnelle » contemporaine, les mooks se revendiquent de la tradition du grand reportage à la française et du narrative writing à l'américaine. Il s'agit donc de revendiquer leur différence, tout en l'inscrivant dans un héritage, une filiation qui lui confère une certaine légitimité historique - avec néanmoins un accent posé différemment pour chaque publication $^{26}$.

21 En effet, avec la multiplication des mooks, les questions d'identité professionnelle semblent devenir plus pointues. Comme l'explique Guillaume Henchoz ${ }^{27}$,

En fait, c'est une mode. Donc évidemment, on y est tous : il y a Gonzaï, XXI, Feuilleton.

On se regarde un peu tous. [...] Puis on se dit: « Mais [...] qu'est-ce qu'ils font ? [...]

ils font comme nous. Qu'est-ce qui nous différencie?» Et puis, ben voilà, quand on

lit des articles sur des blogs ou des journalistes qui disent « ah grand retour du journalisme narratif », on se dit «ah ben voilà, [...] c'est bon, on est déjà étiqueté, on est déjà dans un petit casier quoi. »

Si le modèle narratif reste plus discret dans les pages des quotidiens francophones - jamais identifié en tant que tel, le plus souvent présenté comme "reportage", apparaissant dans les rubriques établies du journal -, il n'en est pas moins tout aussi lié, dans les rédactions de ces journaux, à des enjeux professionnels. Par exemple, Serge Michel explique qu'il a été embauché au Monde, une dizaine d'années auparavant, justement pour ses qualités de conteur :

Cahiers de Narratologie, 31 | 2016 
Le journal avait été orienté très factuel, très journalisme spécialisé, chaque journaliste suivait un domaine très précis. [...] on m'avait dit à peu près « voilà il y a personne dans le journal qui sait raconter une histoire en 10.000 signes. " Et ils avaient créé des formats de 10.000 signes, avec une page portrait, une page récit, etc. et ils avaient de la peine à les remplir. Aujourd'hui, c'est moins le cas et je pense que ça montre qu'il y a eu un rééquilibrage en faveur du narratif là-dessus.

Aux Etats-Unis, le journalisme narratif apparait comme la - ou à tout le moins l'une des marque(s) de fabrique d'un petit nombre de magazines, comme le New Yorker, Harper's ou Esquire. Il s'agit là d'une tradition établie de longue date, qui ne fait plus l'objet d'un discours de différenciation aussi marqué que celui des mooks. Dans les quotidiens ${ }^{28}$, le modèle narratif se présente le plus souvent de manière tout aussi discrète que dans les journaux francophones, sans signes distinctifs quant au genre ou à la rubrique. Certains projets de grande envergure sortent néanmoins de ce cadre, prenant la forme de séries en plusieurs chapitres - on peut noter, au passage, la référence à la littérature dans le choix du terme - ou parties, parfois regroupées dans un cahier spécial entièrement consacré au récit en question. Cependant, aucune mention n'est faite au journalisme narratif dans cette façon de se distinguer du reste de la production du journal.

De manière générale, la conception du modèle narratif en tant que récit long, et donc cher - soit une conception proche de celle que défendent les mooks en Europe francophone -, reste dominante parmi les interviewés américains. Cependant, certains journalistes - comme, on l'a vu, Tom French - appellent à étendre la définition du modèle à des productions plus courtes et réalisées à un rythme plus habituel pour un quotidien. Ainsi, lorsqu'on lui demande de renseigner trois articles particulièrement représentatifs de ce qu'est, selon lui, le journalisme narratif, Mike Wilson ${ }^{29}$ indique un récit long qui s'inscrit dans la conception classique du modèle, mais aussi un récit réalisé le jour des faits pour publication le lendemain - « [it is] in some ways more representative because we do narrative on a daily deadline quite often ${ }^{30}$ ", explique-t-il. Enfin, le troisième récit recommandé est un très court article, composé d'une série de tweets envoyés par un reporter de la rédaction, racontant une petite aventure qui lui est arrivée sur la route, alors qu'il partait couvrir un événement sportif pour le journal - un choix que Mike Wilson justifie ainsi : « Montgomery's story told in tweets I sent as an example of a kind of creative narrative storytelling that some of our most inventive journalists are doing ${ }^{31}$. »

Mike Wilson fait néanmoins figure d'exception parmi les éditeurs américains, et plusieurs journalistes font part, durant les entretiens, de pressions au sein des rédactions à l'encontre des praticiens du modèle narratif. Pourtant, en même temps, les grands projets narratifs permettent aussi aux journaux de se distinguer, notamment en gagnant des prix. Comme l'explique Amy Harmon ${ }^{32}$,

Even though, in the end, I think these stories have high impact and people remember them and they win prizes - one reason I'm allowed to do these stories is because I won a Pulitzer Prize for some of these stories [...] -, there is always tension about doing them. There's always editors who are like "what's taking you so long ${ }^{33}$ ?"

Ce passage montre bien à quel point, dans le modèle journalistique narratif, les considérations éthiques, les questions d'identité professionnelle et les préoccupations économiques constituent des enjeux inextricablement entremêlés. Ces différents enjeux, dans leur enchevêtrement, ne se révèlent pas uniquement dans le discours des praticiens ; ils laissent également des traces dans la poétique des récits journalistiques. 


\section{Une poétique du récit sous influence}

Dans leurs récits, les journalistes narratifs cherchent - consciemment ou inconsciemment - à trouver un équilibre entre plaisir de lecture et confiance du lecteur, attirer et fidéliser, être conteur tout en restant journaliste. Cela se traduit principalement dans la façon dont ils réconcilient, de manière créative et innovante, les fonctions narratives intrigante et configurante. Ces innovations peuvent parfois sembler anodines, parfois frapper par leur originalité. Dans un cas comme dans l'autre, cependant, une analyse détaillée des choix d'écriture des journalistes narratifs, couplée à des entretiens avec ces journalistes, révèle à quel point les innovations formelles, loin d'une « simple » recherche stylistique, sont bien souvent plus complexes qu'elles ne paraissent au premier abord. Cette deuxième partie présente et discute quelques exemples issus d'articles narratifs rédigés ou édités par les praticiens rencontrés en entretiens, tout en les éclairant par les commentaires des journalistes lors de ces entretiens.

\section{Les dessous de l'illusion}

La plupart des articles américains analysés proposent un récit très immersif, avec une intrigue clairement définie, dans lequel le journaliste et ses démarches s'effacent largement, voire totalement, au profit de l'histoire des sujets. Un de ces articles commence par exemple ainsi :

PLANT CITY - The family had lived in the rundown rental house for almost three years when someone first saw a child's face in the window.

A little girl, pale, with dark eyes, lifted a dirty blanket above the broken glass and peered out, one neighbor remembered.

Everyone knew a woman lived in the house with her boyfriend and two adult sons. But they had never seen a child there, had never noticed anyone playing in the overgrown yard.

The girl looked young, 5 or 6 , and thin. Too thin. Her cheeks seemed sunken; her eyes were lost.

The child stared into the square of sunlight, then slipped away ${ }^{34}$.

L'article raconte ensuite comment cette petite fille, négligée par sa mère au point d'être considérée comme une enfant sauvage, est découverte par la police, puis adoptée par une famille qui tente de l'aider à s'adapter à la vie en société. Le récit contient des diagnostics médicaux, des détails intimes, d'autres exemples, historiques et fictionnels, d'enfants sauvages, etc. - le tout présenté avec une autorité qui fait apparaître la journaliste, Lane DeGregory, dans une position assez proche de celle du narrateur omniscient des romans classiques. Cependant, à la fin de l'article, le lecteur trouve l'encadré suivant, intitulé «About this story »:

St. Petersburg Times reporter Lane DeGregory and Times photographer Melissa Lyttle met Danielle and her new family at their home in February. All of the scenes at their house and in speech therapy were witnessed by the journalists.

The opening scene and others were reconstructed from interviews with neighbors, the detective, Danielle's care manager, psychologist, teacher, legal guardian and the 
judge on her case. Additional information came from hundreds of pages of police reports, medical records and court documents.

Michelle Crockett was interviewed at home in Plant City.

In June, Danielle's new parents sold their Florida home and moved out of state.

Bernie built Dani a treehouse. Last week, she began summer school ${ }^{35}$.

Il s'agit là d'une manière, pour le journal, de réaffirmer la qualité de reporter de Lane DeGregory et le caractère réel et vérifié de l'histoire, tout en préservant l'immersion du lecteur dans le récit - ce qui participe à la création d'un autre rapport à l'information et au lecteur. Comme la journaliste l'explique elle-même,

The illusion, if that's the right word, of a narrative is that you're putting the person there. So when you stop and take them out and say "I spent ten hours with this person and we visited nine sites," it removes you from that movie, you know. So I'd rather have like all the credits afterwards or besides or in a little box ${ }^{36}$.

Ce type d'encadré, ou de "boîte » - selon la traduction littérale du terme anglais -, est aujourd'hui un procédé relativement répandu chez les journalistes narratifs américains. Lane DeGregory explique d'ailleurs qu'elle l'a découvert en lisant la série d'articles intitulée «Enrique's journey », de Sonia Nazario, parue dans le Los Angeles Times en 2002 et couronnée par un prix Pulitzer - après plusieurs scandales impliquant des lauréats pour invention ou distorsion des faits. On voit donc bien ici réapparaître des enjeux liés à l'identité professionnelle et à des logiques économiques ${ }^{37}$, au-delà des enjeux éthiques.

Certains journalistes américains - même si c'est rare - décident d'aller plus loin dans la démarche en fournissant, par exemple, un lien vers une page web où sont répertoriées, ligne par ligne, toutes les sources utilisées par le journaliste pour écrire son article ${ }^{38}$. Le journaliste se présente ainsi comme un conteur et, en même temps, presque comme un historien - il est d'ailleurs intéressant de noter que, à l'opposé de cette posture, Léna Mauger, l'une des interviewés francophones, interrogée en entretien sur les sources d'un de ses récits, explique : « Mais bon comme après c'est pas justement une thèse ou un livre, je mettais pas les notes de bas de page pour dire exactement d'où elles [les citations reprises dans l'article] venaient parce que c'est pas le but... c'est pas un livre d'histoire donc..."

A l'autre extrémité du spectre des possibles, il arrive aussi - même si c'est rare également - que la «boîte » devienne le lieu même de l'information, le récit n'étant plus que l'expérience personnelle des sujets dont le journaliste raconte l'histoire. C'est le cas, par exemple, lorsque John Woodrow Cox raconte comment un couple de jeunes parents est informé de la mort par noyade de leur enfant, tout en gardant les statistiques concernant ce type d'accident pour la «boîte » qui accompagne l'article. Si on peut voir dans ce choix une forme - ou, à tout le moins, un risque - de voyeurisme ou de sensationnalisme, ainsi qu'un basculement en faveur de l'identité de conteur par rapport à celle d'informateur, John Woodrow Cox affirme que, pour lui, c'était la meilleure façon d'informer et de conscientiser le lecteur quant à ce sujet : «I was insistent that that story would just be that moment. Because I thought it was so powerful just by itself. It would be more powerful and meaningful that any statistic could be ${ }^{39}$.»

Il existe donc ainsi tout un spectre de figures professionnelles que le journaliste peut créer au travers de son récit. Néanmoins, quelles que soient leurs préférences personnelles, les journalistes narratifs doivent aussi se conformer aux règles et usages en application au sein de leur rédaction : par exemple, alors que le Tampa Bay Times autorise, 
voire parfois encourage, l'utilisation de "boîtes» et d'autres formes de sourçage de l'information, Amy Harmon explique qu'il ne s'agit pas de pratiques qui ont cours au New York Times. La journaliste trouve néanmoins d'autres façons de rappeler au lecteur sa qualité de journaliste, sans pour autant briser ses effets narratifs en tant que conteuse. Par exemple, dans un portrait d'un jeune autiste prénommé Justin, dont elle raconte le combat pour trouver un travail, en adoptant une position de narration très largement empathique par rapport à son protagoniste, la journaliste note à l'occasion : « He bristled at criticism, particularly of his artwork ("No opinions, please" was a Justinism his teachers knew well ${ }^{40}$ ). " Alors qu'on pourrait ne voir dans cette touche d'humour qu'un petit plaisir textuel glissé pour le lecteur, ou la marque de fabrique de l'auteur ou du titre de presse qui tente ainsi de se distinguer de la masse de ses concurrents, Amy Harmon explique qu'il s'agit pour elle de montrer à son lecteur qu'il peut lui faire confiance, qu'elle conserve, malgré toute son empathie, une certaine distance critique pour raconter cette histoire :

I guess I'm just trying to be like a kind observer, a sympathetic observer with a little bit of a sense of humor. That's who I would kind of trust, I guess, to tell a story. I'm not trying to be totally objective. In any story like this you're a sympathetic ear but with enough of a sense of humor and of perspective on your character. Those moments where I'm gently making fun, it's important to do that so that the reader knows you're not just completely bought into this character ${ }^{41}$.

\section{Jeux de personnes}

Contrairement aux Américains, les praticiens francophones rencontrés ont tendance à être présents, de manière plus ou moins affichée, dans leurs récits. Les démarches journalistiques font ainsi, dans une certaine mesure, partie même du récit. Par ailleurs, si une volonté de "tenir le lecteur en haleine»-pour reprendre l'expression de Léna Mauger et de Serge Enderlin ${ }^{42}$ - guide bien l'organisation du récit, les journalistes francophones insistent plus sur les idées de qualité de l'écriture ou de style que sur celles de tension ou de suspense. La question du rapport entre plaisir de lecture et confiance du lecteur se pose donc quelque peu différemment dans ces textes, entraînant des réponses forcément différentes également sur le plan poétique.

Il existe de nombreuses façons, pour un journaliste, de marquer sa présence au sein de son récit. Les interviewés francophones jouent sur une large gamme qui va de l'apparente indétermination du on à l'affichage explicite d'un je, en passant notamment par le nous d'auteur. Si ces choix constituent incontestablement des partis pris assez rares en journalisme, ils n'apparaissent pas pour autant comme réellement novateurs. Certains textes font néanmoins preuve de plus d'originalité dans leur utilisation des pronoms personnels, par exemple en mettant en œuvre un je plus singulier que les autres - et que l'on pourrait qualifier, à la suite du journal Ithaque, d'« introspectif ${ }^{43}$ ». Il ne s'agit pas uniquement, comme dans les autres récits francophones à la première personne, de rappeler la présence du journaliste sur le terrain, d'expliciter les démarches de reportage ou d'enquête, ni même de partager à l'occasion quelques réactions plus personnelles aux événements dont le journaliste a été témoin. Le je est également utilisé, dans ces quelques textes, pour expliquer l'origine de l'article, mettre à plat la relation du journaliste à son sujet, faire part de ses opinions plus personnelles, etc. - par exemple :

Je le regarde écouter Kasparov. Ronger son frein. Effiler les pointes de sa moustache

d'un geste qui ressemble à un tic, et qui me déplaît. Je me demande ce qu'il pense, 
ce qu'il espère. Est-ce qu'il y croit ? Est-ce que ça l'amuse, de jouer pour un temps ce rôle d'homme politique à peu près respectable, lui l'outlaw, le chien enragé ? Est-ce une ruse tactique ${ }^{44}$ ? faire de lien entre l'originalité de cette posture et le fait que l'article ait été récompensé par le prix Albert Londres - offrant ainsi une certaine légitimité et une certaine publicité non seulement à la jeune journaliste, mais aussi à la jeune revue. D'un point de vue éthique, on peut voir dans ce choix de pronoms une façon pour Sophie Bouillon d'indiquer au lecteur que, même si elle va tenter de lui faire comprendre au plus près le point de vue de ce jeune homme, elle reste la narratrice de cette histoire, et donc le filtre indépassable au travers duquel le lecteur découvre le protagoniste. Comme elle l'explique en entretien : «c'est une interprétation, c'est comme quand on va voir un psy en fait un peu, qui est en train de décrire, de mettre en mots ce qu'il a perçu chez vous. »

Cependant, en entretien, la journaliste fait également part d'une réelle inquiétude par rapport à ses choix stylistiques - une inquiétude qui renvoie, encore une fois, tant à des considérations éthiques qu'à des questions d'identité professionnelle :

Là, je vais prendre vraiment l'exemple de «Bienvenue chez Mugabe!» parce que c'est le plus narratif dans le sens où c'est le plus proche de l'écriture littéraire. Ce qui m'inquiétait vachement, dans ce texte, c'est que les gens pensent que ce soit une fiction, c'est que ce soit tellement éloigné en fait du style journalistique qu'on se dise que c'est une belle histoire quoi, qu'on se dise que c'est bien écrit, c'est joli 
[...] et moi, des fois, ce qui me fait peur là-dedans, c'est qu'on ait tendance à lire le journalisme comme quelque chose qui n'existe pas vraiment.

\section{Aux frontières de l'expérimentation}

43 Ce risque, inhérent à la définition même du journalisme narratif, semble d'autant plus prononcé que le journaliste s'écarte de la poétique de l'article de presse "classique ", qu'il en interroge et repousse les frontières. Cela n'empêche néanmoins pas certains journalistes et certains médias de pousser plus loin leurs expérimentations avec la forme narrative. Le récit plus innovant, sur l'ensemble des articles analysés, est l'article en tweets déjà mentionné et recommandé pour l'analyse par le rédacteur en chef du Tampa Bay Times en ce qu'il est justement un exemple d'un type de récit créatif produit par l'un des journalistes les plus inventifs de la rédaction. L'article est introduit comme suit :

On his way to cover a baseball game in Miami on Oct. 2, Ben Montgomery made an unexpected stop by the side of the road. He wrote about what happened in a series

of 15 tweets. Here they are exactly as they appeared, beginning at 9:46 a.m $\mathrm{m}^{48}$.

La suite de l'article n'est que la série de tweets du journaliste, confronté à ce qui pourrait être un guet-apens pour le voler :

1. Driving south toward Miami when I see a pregnant woman on the shoulder by a

s----- brown mini van. She's waving her arms. [...]

7. I'm fully freaked, but maybe it's not what it seems. [...]

12. I'm feeling very much alive. No idea if it was a trap ${ }^{49}$.

Le contenu informatif de l'article est extrêmement pauvre ${ }^{50}$, mais l'article recrée la tension - et donc le plaisir qui y est associé - vécue par les lecteurs des tweets à l'origine. Ici, les logiques économiques et professionnelles semblent très présentes : mise en avant d'un journaliste du quotidien, connu et reconnu pour ses articles narratifs, dans une petite histoire dont le but principal semble être le plaisir de lecture - selon l'explication de Mike Wilson, en lisant ces tweets, "[Montgomery's] editors and his other colleagues [...] said "oh my gosh, that's a story right there." [...]. It was just great reading ${ }^{51}$.» Le quotidien affiche ainsi la qualité de conteur d'un des reporters de l'équipe, ainsi que ses qualités humaines - le titre de l'article fait référence au «bon samaritain »-, tout en exposant une certaine créativité du journal.

Pourtant, il y a quand même un certain message, une interrogation fondamentale sur nos sociétés dans cette petite histoire, comme on le voit dans le dernier tweet : «And then the question: Is it better to lend a hand - with risk - or stay in the line of the impartial. I don't $\mathrm{know}^{52}$.» Comme l'explique encore Mike Wilson, la fonction première de cette histoire n'était pas vraiment d'informer, mais plutôt de décrire une expérience que les lecteurs pourraient comprendre et par laquelle ils pourraient se sentir concernés. S'il ne s'agit pas là de la visée considérée généralement comme première en journalisme, elle n'en relève pas moins - comme on l'a vu au travers des propos de Patrick de Saint-Exupéry et de la notion d'identité narrative -, de considérations éthiques fondamentales.

\section{Conclusion}

Ce dernier exemple illustre donc à nouveau à quel point les enjeux éthiques - dans le sens restreint de l'éthique journalistique, tout comme dans un sens plus large -, économiques 
et professionnels s'imbriquent les uns dans les autres, tout en s'avérant inséparables de la dimension formelle et poétique du récit journalistique. Si l'analyse d'un récit de fiction gagne bien entendu à tenir compte du contexte de production du récit, cette prise en compte apparaît donc, dans le cadre des récits journalistiques, absolument nécessaire. Or, parce que le journalisme narratif constitue un modèle alternatif au modèle dominant, le contexte de production de ces récits diffère du contexte de production des articles de presse «classiques ». C'est en comprenant les visées éthiques propres au modèle, les enjeux économiques qui s'y jouent et les questions d'identité professionnelle qu'il soulève - notamment - que l'on peut pleinement comprendre les choix stylistiques et poétiques des journalistes narratifs. Sans prétendre tracer un tableau complet des enjeux contextuels propres au modèle narratif, cet article permet de poser les bases d'une approche poétique et en contexte du journalisme narratif.

Il apparait toutefois tout aussi important, dans un second temps, de s'intéresser au contexte de réception des articles narratifs et de se demander, au-delà de l'analyse textuelle et au-delà des discours des praticiens, comment les récits journalistiques sont effectivement reçus et perçus par les lecteurs. Offrent-ils réellement un plus grand plaisir de lecture et une meilleure compréhension de l'information, comme leurs auteurs le soutiennent? Sur quoi reposent ce plaisir de lecture et cette compréhension? Et comment, dès lors, ces récits modifient-ils le rapport des lecteurs aux journalistes et aux médias qui les mettent en œuvre ? Il s'agit là d'autant de pistes à explorer dans de futures recherches.

Enfin, dans une perspective plus large, les contextes de production et de réception étant fondamentalement différents dans le cas d'un roman, par exemple, et dans celui d'un article narratif, l'étude des récits propres au modèle journalistique narratif semble pouvoir s'avérer particulièrement féconde pour la narratologie. Comme on l'a montré dans cet article, des contraintes, des visées, des effets - réels ou espérés - différents impliquent, assez logiquement, une poétique au moins en partie différente. La narratologie ne peut que s'enrichir au contact de récits qui sortent de son domaine d'analyse privilégié - celui de la fiction. C'est une voie déjà empruntée par de nombreux travaux au sein de ce que l'on a appelé la narratologie médiatique (notamment Lits, 1997b, 2008 ; Marion, 1997 ; Revaz, 1997 ; Revaz, Pahud et Baroni, 2007). Toutefois, en se situant à mi-chemin entre le récit de fiction et le récit médiatique "classique», le journalisme narratif se présente comme un terrain d'étude particulièrement intéressant pour faire se rencontrer et dialoguer divers outils, concepts et courants narratologiques.

\section{BIBLIOGRAPHY}

ADAM J.-M., REVAZ F., 1996, L'analyse des récits, Paris, Seuil.

BARONI R., 2007, La tension narrative : Suspense, curiosité et surprise, Paris, Seuil.

BARONI R., 2008, « Approches passionnelles et dialogiques de la narrativité », Cahiers de narratologie , 14 . 
BARONI R., 2009, L'œuvre du temps : Poétique de la discordance narrative, Paris, Seuil.

CORNU D., 2009, Journalisme et vérité : L'éthique de l'information au défi du changement médiatique, Genève, Labor et Fides.

CRAIG D., 2006, The Ethics of the Story: Using Narrative Techniques Responsibly in Journalism, Lanham, Rowman \& Littlefield.

FRANKLIN J., 2002, Writing for Story : Craft Secrets of Dramatic Nonfiction by a Two-Time Pulitzer Prize Winner, New York, Plume.

KRAMER M., 1995, « Breakable Rules for Literary Journalists », dans SIMS N., KRAMER M. (dirs.), Literary Journalism : A new collection of the best American nonfiction, New York, Ballantine Books, p. 21-34.

LALLEMAND A., 2011, Journalisme narratif en pratique, Bruxelles, De Boeck.

LITS M., 1997a, « Le récit médiatique : un oxymore programmatique? », Recherches en

Communication, 7, p. 36-59.

LITS, M. (dir.), 1997b, Recherches en communication, $n^{\circ} 7$ : Le récit médiatique.

LITS M., 2008, Du récit au récit médiatique, Bruxelles, De Boeck.

LITS M., 2010, «L'impossible clôture des récits multimédiatiques », A contrario, 13, p. 113-124.

LITS M., 2012, « Quel futur pour le récit médiatique ? », Questions de communication, 21, p. 37-48.

MARION P., 1997, « Narratologie médiatique et médiagénie des récits », Recherches en Communication , 7, p. 61-88.

MOSSER J., 2012, « What's gonzo about Gonzo Journalism ? », Literary Journalism Studies, 4, 1, p. 85-90.

REVAZ F., 1997, « Le récit dans la presse écrite », Pratiques, 94, p. 19-33.

REVAZ F., PAHUD S., BARONI R., 2007, « Classer les “récits” médiatiques : entre narrations ponctuelles et narrations sérielles ", dans CHRÄ̈BI A. (dir.), Classer les récits : théories et pratiques, Paris, L'Harmattan, p. 59-82.

RICœUR P., 1990, Soi-même comme un autre, Paris, Seuil.

RICœUR P., 1991, Temps et récit, tome 1 : L'intrigue et le récit historique, Paris, Seuil (Points).

sCHUDSON M., 1978, Discovering the News : A Social History of American Newspapers, New York, Basic Books.

STEWART J., 1998, Follow the Story : How to Write Successful Nonfiction, New York, Simon \& Schuster. VANOOST M., 2013a, « Defining narrative journalism through the notion of plot », Diegesis, 2, 2, p. 77-97.

VANOOST M., 2013b, « Journalisme narratif : proposition de définition, entre narratologie et éthique », Les Cahiers du journalisme, 25, p. 140-161.

WEINGARTEN M., 2006, The gang that wouldn't write straight, New York, Crown Publishers.

WOLFE, T. (dir.), 1975, The New Journalism, New York, Harper \& Row. 


\section{NOTES}

1. Nos italiques.

2. Au sens de l'opération de configuration ricœurienne, au travers de laquelle les différent éléments qui composent une histoire sont « pris ensemble ", de manière à réaliser une « synthèse de l'hétérogène » (Ricœur, 1991, p. 127).

3. Voire même un degré de configuration plus élevé, comme on le verra un peu plus loin.

4. Le terme intrigue étant pris ici au sens que lui donne Baroni (2007), à la suite notamment de Jean-Michel Adam et Françoise Revaz (1996), soit la dynamique tensive qui se crée entre un nœud au début du récit et la promesse de son dénouement à la fin du texte.

5. Les entretiens avaient pour but premier de discuter des pratiques narratives de ces journalistes, principalement sous un angle éthique. Ils ont néanmoins fait apparaître également des questions économiques et des logiques identitaires.

6. Un mot formé à partir des termes anglais magazine et book pour désigner d'épaisses revues au rythme de parution lent, privilégiant des articles longs, dépourvues de publicité et dont le prix avoisine celui d'un livre à couverture rigide. Après la pionnière revue XXI, plusieurs autres mooks ont vu le jour : Feuilleton, Desports, Long Cours, 24h01, etc.

7. Pour reprendre les termes utilisés par David Craig (2006, p. 29) pour les deux volets de son analyse éthique des techniques d'écriture journalistique.

8. Journaliste au Tampa Bay Times au moment de l'entretien (le 19 avril 2013).

9. «De nombreuses histoires comportent ce que j'appelle du brocoli. Le brocoli, c'est quelque chose qui est bon pour nous. Il faut en manger. Peut-être qu'on ne l'aime pas, mais on devrait en manger, c'est bon pour nous. Dans une histoire, ça peut être une explication technique, des chiffres, un élément scientifique ou que sais-je encore. Autant de choses que les lecteurs n'ont pas envie de lire, qui leur font perdre patience. [...] Vous avez aussi besoin de cornets de crème glacée pour rendre le brocoli tolérable. Et ces cornets de glace deviennent alors la force qui guide le lecteur à travers l'histoire " (notre traduction, comme pour tous les extraits d'entretiens et d'articles suivants).

10. Journaliste puis éditrice dans différents journaux aux Etats-Unis, même si l'entretien (réalisé le 13 mars 2013) a principalement porté sur son travail au St. Paul Pioneer Press.

11. Fondateur et rédacteur en chef de XXI (entretien réalisé le 28 janvier 2013).

12. Journaliste au Washington Post (entretien réalisé le 15 avril 2013).

13. «Je pense que l'un des plus grands dilemmes éthiques, dans ce type d'écriture, est de dissimuler certaines informations au lecteur, de le manipuler, en quelque sorte, en dévoilant certaines informations à la fin, lors de la résolution. D'une certaine manière, c'est l'inverse même de tout ce que l'on apprend quand on devient journaliste. Et je n'ai pas vraiment de réponse à ce problème. Je l'ai fait moi-même. Oui, c'est quelque chose qu'on fait, je le fais dans presque toutes mes grosses histoires, parce qu'il faut qu'il y ait une raison qui pousse le lecteur à continuer. »

14. Anciennement journaliste au Tampa Bay Times (entretien réalisé le 25 mars 2013).

15. «D'une certaine manière, il se pourrait que nous soyons même un peu trop programmés pour le récit, parce que les histoires peuvent complètement façonner et influencer la manière dont nous concevons l'Histoire. Ce sont les conteurs qui détiennent notre vision de l'Histoire. Cette idée selon laquelle, si vous possédez le droit de raconter l'histoire, vous possédez en quelque sorte aussi la vérité, waouw, c'est une responsabilité énorme. "

16. Journaliste au Washington Post (entretien réalisé le 16 avril 2013).

17. «Il me semble que, d'une part, c'est une technique qui relève de l'instinct de survie pour les journalistes et les journaux. Je pense qu'il faut faire en sorte que les lecteurs se sentent concernés 
pour qu'ils s'investissent dans ce que l'on écrit. Mais je pense aussi qu'il n'y a pas de meilleure façon de comprendre ce que les gens vivent et à quoi ressemblent leurs vies. »

18. Journaliste au Tampa Bay Times au moment de l'entretien (réalisé le 21 mars 2013).

19. "Je pense que c'est vraiment la seule façon... ou en tout cas c'est une façon pour les journaux de survivre. Je pense que les journaux doivent apporter une valeur ajoutée à la vie des gens et qu'une façon de le faire est de leur donner de très bonnes histoires à lire. »

20. Journaliste et adjointe à la rédaction en chef de XXI (entretien réalisé le 9 juillet 2013).

21. Journaliste au Tampa Bay Times (entretien réalisé le 1 mars 2013).

22. "Quand les éditeurs reçoivent la mise en page du cahier réservé aux features, ils viennent me dire: "Tu as six pouces [quinze centimètres] sur la Une." Et donc je me dis : "A la fin de ces six pouces, il faut que je place un bon élément, comme une question, pour que le lecteur doive tourner la page." C'est une chose à laquelle je pense vraiment ; c'est comme si je traçais un chemin avec des miettes de pain, en espérant que le lecteur les ramasse et continue à suivre l'histoire. "

23. Directeur de publication de Feuilleton et Desports (entretien réalisé le 6 juin 2013).

24. Même s'il convient de souligner ici que certains titres sont revenus à des formules plus classiques, comme Usbek et Rica, ou ont tout bonnement vu leur publication prendre fin, comme Alibi ou Long Cours.

25. «Je pense qu'il faut aider les gens à élargir leur définition de ce qu'est un récit. Beaucoup d'éditeurs et journalistes considèrent que seuls les très longs reportages de fond peuvent prendre la forme d'un récit. »

26. Ainsi, pour Patrick de Saint-Exupéry et XXI, « la base du journalisme narratif, c'est Albert Londres, c'est Kessel. Enfin voilà, ça vient d'Europe, ensuite c'est reparti aux Etats-Unis, où là ça $a$ été transformé en narrative writing et, comme ça vient des Etats-Unis, ça revient en Europe, mais à l'origine, c'est toujours venu d'ici." Pour Adrien Bosc et Feuilleton, les racines sont plutôt américaines, avec «une tradition qui n'existe pas ailleurs, ou très peu. » A la barre d'Ithaque, Guillaume Henchoz pointe plus spécifiquement vers le gonzo journalism américain (Mosser, 2012 ; Weingarten, 2006).

27. Rédacteur en chef d'Ithaque au moment de l'entretien (réalisé le 26 juin 2013).

28. Où travaillent la plupart des praticiens américains interviewés ici.

29. Rédacteur en chef du Tampa Bay Times au moment de l'entretien (réalisé le 8 mars 2013).

30. «D'une certaine façon, c'est plus représentatif parce que nous faisons assez souvent du récit à un rythme quotidien. "

31. "L'article en tweets de Montgomery, je l'ai envoyé en tant qu'exemple du genre de récits créatifs que nos journalistes les plus inventifs expérimentent. »

32. Journaliste au New York Times (entretien réalisé le 10 avril 2013).

33. "Même si je pense qu'au final ces histoires ont un impact fort, que les gens s'en souviennent et qu'elles gagnent des prix - l'une des raisons pour lesquelles je peux faire ce type de journalisme, c'est que j'ai gagné un prix Pulitzer pour certaines de ces histoires -, écrire ce genre d'histoires ne va jamais sans tension. Il y a toujours des éditeurs pour nous demander ce qui nous prend tant de temps. »

34. Lane DeGregory, «The girl in the window », The Tampa Bay Times, 31 juillet 2008.

«PLANT CITY - La famille s'était installée dans la maison décrépite depuis presque trois ans quand quelqu'un remarqua pour la première fois le visage d'une enfant à la fenêtre.

Une petite fille, pâle, aux yeux sombres, souleva une couverture crasseuse au-dessus de la vitre cassée et jeta un coup d'œil à l'extérieur, se souvient un voisin.

Tout le monde savait qu'une femme vivait dans la maison avec son compagnon et ses deux fils adultes. Mais personne n'avait jamais vu une enfant là, n'avait jamais remarqué quelqu'un jouer 
dans le jardin envahi par la végétation.

La petite fille avait l'air jeune, 5 ou 6 ans, et maigre. Trop maigre. Ses joues semblaient creuses, ses yeux égarés.

L'enfant regarda fixement le carré de lumière que dessinait le soleil, puis disparut. »

35. «La reporter Lane DeGregory et la photographe Melissa Lyttle, du Saint Petersburg Times, ont rencontré Danielle et sa nouvelle famille à leur domicile en février. Elles ont assisté à toutes les scènes qui prennent place chez eux et lors des séances de thérapie du langage.

La scène d'ouverture, ainsi que d'autres, ont été reconstituées à partir d'interviews avec des voisins, l'inspecteur de police, la gestionnaire de soins de Danielle, sa psychologue, son institutrice, son tuteur légal et le juge qui s'est occupé de son cas. Des informations additionnelles proviennent de centaines de pages de rapports de police, de dossiers médicaux et de documents du tribunal.

Michelle Crockett a été interviewée à son domicile, à Plant City.

En juin, les nouveaux parents de Danielle ont vendu leur maison en Floride et ont déménagé dans un autre état. Bernie a construit une cabane dans un arbre pour Dani. La semaine passée, elle a commencé l'école d'été. »

36. «L'illusion, si c'est le mot juste, d'un récit est de placer le lecteur là [où se déroule l'histoire]. Donc quand vous vous arrêtez pour dire “j'ai passé dix heures avec cette personne et nous nous sommes rendus dans neuf endroits", ça vous fait sortir du film. C'est pour ça que je préfère regrouper tous ces informations après, ou à côté, ou dans un petit encadré. »

37. La crise économique que traversent les journaux étant notamment liée à une crise de confiance des lecteurs.

38. Par exemple : Ben Montgomery, «Spectacle : The lynching of Claude Neal», The Tampa Bay Times, 20 octobre 2011.

39. «J'ai insisté pour que cette histoire ne raconte que ce moment. Parce que je pensais que c'était plus fort ainsi. C'était plus fort et plus significatif qu'aucune statistique n'aurait pu l'être. » 40. "Il se hérissait à la critique, particulièrement de ces dessins ("Pas d'avis, s'il vous plaît" était un Justinisme que ses professeurs avaient l'habitude d'entendre). »

41. «Je suppose que j'essaie de me mettre dans la peau d'une observatrice gentille, sympathique, avec une touche de sens de l'humour. Il me semble que c'est le genre de narrateur auquel je ferais confiance. Je n'essaie pas d'être entièrement objective. Dans n'importe quel récit de ce genre, vous êtes une oreille bienveillante, mais avec suffisamment de sens de l'humour et de perspective par rapport à votre personnage. Ces moments où je rigole gentiment sont importants pour montrer au lecteur que vous gardez un certain esprit critique par rapport au personnage. »

42. Journaliste à 24 Heures, même si l'entretien (réalisé le 24 juin 2013) a principalement porté sur son travail pour Le Temps.

43. Ithaque identifie notamment un texte de Guillaume Henchoz (« La route de la viande », Ithaque , hiver 2012) comme « reportage introspectif».

44. Emmanuel Carrère, « Le dernier des possédés », XXI, hiver 2008.

45. Laurent Beccaria et Patrick de Saint-Exupéry, « Editorial », XXI, hiver 2008, p. 3.

46. Journaliste indépendante, qui a travaillé notamment pour Libération et XXI (entretien réalisé le 21 juillet 2013).

47. Sophie Bouillon, «Bienvenue chez Mugabe! », XXI, automne 2008.

48. Ben Montgomery, "Word for word: The Samaritan considers his options ", The Tampa Bay Times, 30 novembre 2012. 
«En route pour couvrir un match de baseball à Miami le 2 octobre, Ben Montgomery a fait un arrêt imprévu sur le bord de la route. Il a décrit ce qui s'est passé dans une série de 15 tweets. Les voici exactement comme ils ont été publiés, en commençant à 9h46. »

49. «1. Je conduis vers le sud, en direction de Miami, quand je vois une femme enceinte sur la bande d'arrêt d'urgence, à côté d'un mini van brun. Elle agite les bras. [...]

7. Je flippe complètement, mais peut-être n'est-ce pas ce que ça semble être. [...]

12. Je me sens tellement vivant. Impossible de dire si c'était un piège. "

50. Il s'agit d'une anecdote personnelle, sans noms, sans localisation précise, sans information visant une contextualisation plus large.

51. "Les éditeurs et collègues de Montgomery se sont dits "oh mon dieu, c'est un récit ça." C'était juste tellement agréable à lire. »

52. «Et puis la question : Est-ce mieux de tendre la main - et de prendre un risque - ou de rester indifférent ? Je ne sais pas. »

\section{ABSTRACTS}

As a hybrid model halfway between journalism and literature, narrative journalism lies at the heart of a complex web of ethical, economic and professional issues - notably - that weigh on its poetics. This paper first explores the inescapable entanglement of these different kinds of issues. It then illustrates, through a few examples, how this complex network gives rise to innovations in the narrative form. These innovations aim at creating a reading experience close to the experience of fiction reading while reaffirming the nonfiction status of the narrative, at attracting readers but also at gaining their loyalty, at constructing a professional figure as both a storyteller and a journalist.

Modèle hybride entre journalisme et littérature, le journalisme narratif se situe au cœur d'enjeux éthiques, économiques et professionnels - notamment - qui pèsent sur sa poétique. Le but de cet article est d'explorer l'inévitable enchevêtrement de ces enjeux d'ordres différents et de montrer, à partir de quelques exemples, comment cet enchevêtrement donne naissance à des innovations sur le plan formel, visant à rapprocher l'expérience de lecture de celle d'un récit de fiction tout en rappelant le caractère réel du récit, à attirer puis fidéliser le lecteur, à se présenter à la fois comme un conteur et un journaliste.

\section{INDEX}

Mots-clés: journalisme, récit, contexte de production, poétique, éthique, économie, identité professionnelle

Chronological index: XXe siècle, XXIe siècle

Geographical index: Europe francophone, Etats-Unis 


\section{AUTHOR}

\section{MARIE VANOOST}

University of North Carolina, School of Media and Journalism 